\title{
Homeopathic Potencies May Possess an Electric Field(-like) Component: Evidence from the Use of Encapsulated Solvatochromic Dyes
}

\author{
Steven J. Cartwright ${ }^{1}$ \\ ${ }^{1}$ DiagnOx Laboratory, Cherwell Innovation Centre, Upper Heyford, \\ Oxon, United Kingdom
}

Homeopathy 2020;109:14-22.

\author{
Address for correspondence Steven J. Cartwright, PhD, DiagnOx \\ Laboratory, Cherwell Innovation Centre, Upper Heyford, Oxon, \\ OX25 5HD, United Kingdom \\ (e-mail: steven.cartwright@oxford-homeopathy.org.uk).
}

\begin{abstract}
Keywords

- solvatochromic dyes

- $\beta$-cyclodextrin

- Arsenicum 10M

- electric field

- metastable state

Background Homeopathic potencies have been shown to interact with a range of solvatochromic dyes to produce spectroscopic changes in the visible region of the electromagnetic spectrum. Furthermore, the nature of the changes observed under different experimental conditions is beginning to limit the number of possible hypotheses that can be put forward regarding the fundamental identity of potencies. Aims and Methods The present study uses $\beta$-cyclodextrins to encapsulate solvatochromic dyes of widely varying structures. The purpose of this approach is to de-couple the primary dye-potency interaction from any subsequent aggregation effects.

Results Despite large differences in molecular structure between dyes, results show that potencies affect all dyes according to the same fundamental principles. Specifically, positively and negatively solvatochromic dyes collectively respond in opposite and complementary ways to potencies in accordance with the differential stabilisation of their excited and ground electronic states. Under the conditions of encapsulation, positively solvatochromic dyes display a bathochromic shift of, on average, $0.4 \mathrm{~nm}$ with a $2 \%$ absorbance change, and negatively solvatochromic dyes display a hypsochromic shift of, on average, $0.2 \mathrm{~nm}$ with a $1 \%$ absorbance change. This behaviour is only ever seen in two situationswhere solvent becomes more polar or where an electric field is applied to solutions of dyes. Conclusions The conditions used in this and previous studies to investigate the interaction of potencies with solvatochromic dyes preclude increased polarity of solvent as being responsible for the observed effects and that an explanation in which potencies carry an electric field (or electric field-like) component is by far the more likely. From the magnitude of the spectral changes induced in the dye Brooker's merocyanine by Arsenicum 10M, an estimate of the strength of the postulated electric field of $1.16 \times 10^{7} \mathrm{~V} / \mathrm{m}$ can be made, which is comparable with the potential difference across cell membranes.
\end{abstract}

\section{Introduction}

Several studies have shown that homeopathic potencies interact with a large range of $\pi$-conjugated push-pull, or solvatochromic, molecular systems. ${ }^{1-4}$ This interaction produces changes in the dyes' visible spectrum, which can be

received

May 1, 2019

accepted after revision

May 30, 2019

published online

August 27, 2019

monitored over time and provides information about potencies at an existential level.

'What are homeopathic potencies?' and 'how do they act to produce clinical effects?' are probably the two most important questions that can be asked in the field of homeopathy. The

Copyright @ 2020 The Faculty of Homeopathy
DOI https://doi.org/ 10.1055/s-0039-1693985. ISSN $1475-4916$. 
current study aims to address these questions and provide the beginnings of an answer by separating out the initial dyepotency interaction free of any subsequent solute and solventmediated effects. Studies so far have shown that the spectral changes seen with solvatochromic dyes in the presence of potencies are the combination of a primary dye-potency interaction followed by subsequent dye $\mathrm{p} K_{\mathrm{a}}$ changes and dye aggregation processes. ${ }^{3}$ To understand more about how potencies are acting, it is therefore important to de-couple the primary interaction from these latter consequential effects.

Encapsulating solvatochromic dyes with cyclodextrins excludes solvent-that is, water ${ }^{5}$-from the vicinity of dyes and prohibits dye aggregation. ${ }^{6}$ By achieving this, the effect of potency on the dye intramolecular charge transfer process $^{7}$ can be examined in detail and any extraneous complicating processes removed. In other words, it is possible to study the initial dye-potency interaction in its pure form.

The structure of a typical cyclodextrin, $\beta$-cyclodextrin sulphobutylether ( $\beta$-CDSBE) is given in - Fig. 1, together with a schematic representation showing the hydrophilic exterior and hydrophobic (lipophilic) core in which dyes are held. ${ }^{8}$ Brooker's merocyanine (BM), as a representative dye, is also shown for size comparison. On encapsulation, the dye moves into the interior of the cyclodextrin. Encapsulation occurs spontaneously on adding dyes to solutions of cyclodextrins, and the choice of which type of cyclodextrin to use
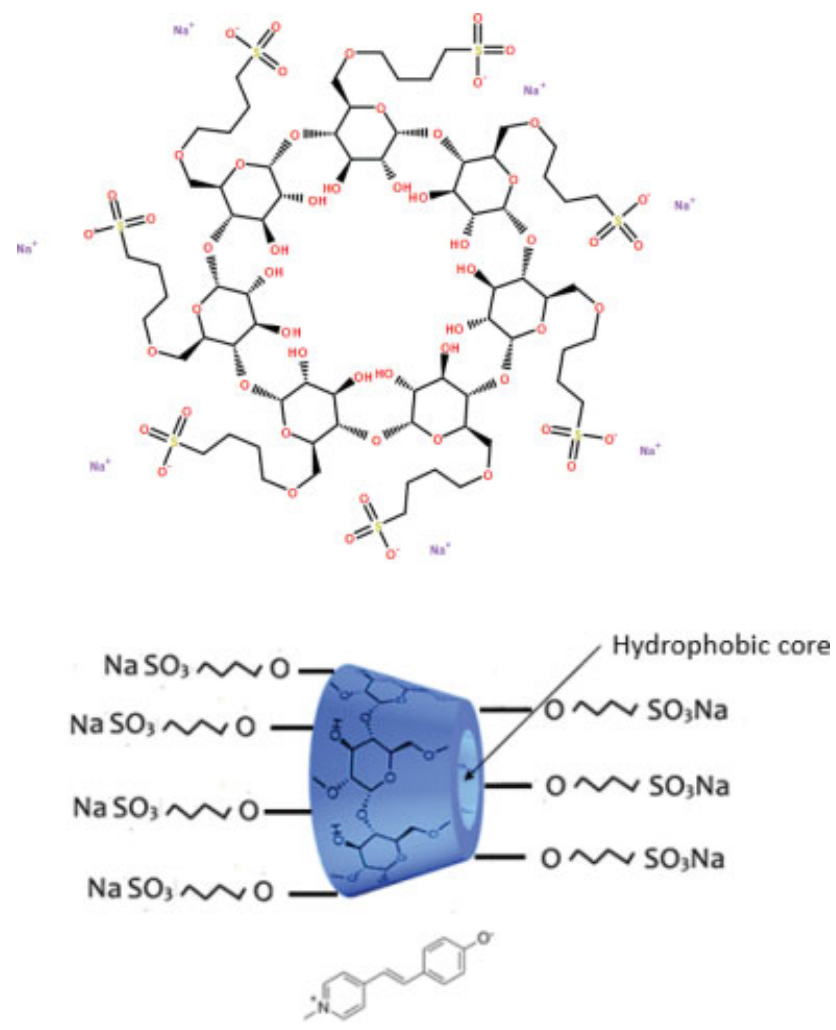

Fig. 1 Molecular structure of $\beta$-cyclodextrin sulphobutylether (top); schematic representation of $\beta$-cyclodextrin sulphobutylether (bottom) showing the hydrophobic interior of the encapsulator in which dyes are bound, together with the structure of dye Brooker's merocyanine for comparison.
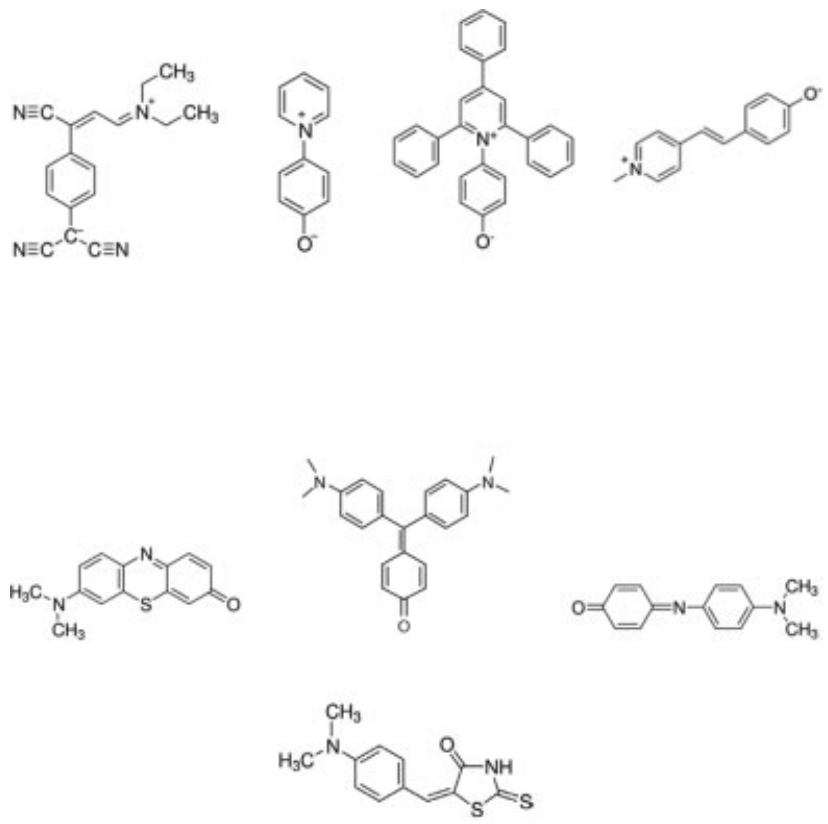

Fig. 2 Structures of dyes used in this study. From left to right (upper panel)-negatively solvatochromic dyes cyano-3-[4-(dicyanomethyl) phenyl]-2-propenylidene]- $N$-ethyl-ethaniminium inner salt (DEMI), 4pyridinium phenolate (4PP), 4-(2,4,6-triphenylpyridinium-1-yl) phenolate (ET1), Brooker's merocyanine (BM); from left to right (lower panel)-positively solvatochromic dyes methylene violet (Bernthsen) $(\mathrm{MV})$, bis-dimethylaminofuchsone (BDF), phenol blue (PB), dimethylamino-benzylidene-rhodanine (DMABR).

is made on the basis of dye structure. The dyes used in the current study are shown in -Fig. 2. Bulky dyes such as bisdimethylaminofuchsone (BDF), cyano-3-[4-(dicyanomethyl) phenyl]-2-propenylidene]- $N$-ethyl-ethaniminium inner salt (DEMI), and 4-(2,4,6-triphenylpyridinium-1-yl) phenolate (ET1) require $\beta$-cyclodextrin sulphobutylether ( $\beta$-CDSBE), whereas phenol blue (PB) can be accommodated by $\alpha$ cyclodextrin $(\alpha-C D)$, which has a smaller encapsulator core diameter.

Many dyes can be accommodated by $\beta$-cyclodextrin ( $\beta-C D)$, which lacks sulphobutylether arms. Binding constants for dyes by $\beta-C D$ typically lie in the range 200 to $5000 \mathrm{M}^{-1}$. Examples include: $\mathrm{BM}, 430 \mathrm{M}^{-1}$; Crystal Violet, which has a similar structure to BDF, $2630 \mathrm{M}^{-1}$; Oxazine 1 , which has a similar structure to Methylene Violet (MV), $1120 \mathrm{M}^{-1}$; and dimethylamino-phenyl-methylene-barbituric acid, similar in structure to dimethylamino-benzylidene-rhodanine (DMABR), $1000 \mathrm{M}^{-1}{ }^{9-12}$ Binding constants for $\beta$-CDSBE and dyes are generally 5 to 10 times higher (i.e. more tightly bound) than for $\beta$-CD. ${ }^{13,14}$ Based on the above estimates and under the conditions used in this study, the percentage of total dye encapsulated should be $\geq 90 \%$ with $\beta$-CD and $\geq 99 \%$ with $\beta$-CDSBE. Hence any spectral changes seen under the action of potency can be attributed to encapsulated dye.

Encapsulation also has several other advantages beyond excluding solvent and suppressing dye aggregation. Poorly water-soluble dyes are solubilised by cyclodextrins ${ }^{15}$ and dyes that are sensitive to light, oxygen or water degradation 
are protected. This means that dyes such as DEMI ${ }^{16}$ that are both poorly water soluble and sensitive to decomposition can be examined, together with PB and ET1, which have low water solubility. The more water soluble and stable dyes that have been examined previously include BDF, 4-pyridinium phenolate (4PP), BM and MV.,3 DMABR and DEMI have not been examined before and provide significantly different structures from previously studied dyes (-Fig. 2).

The dyes used in the current study were chosen on the basis of their type of solvatochromism and to represent as wide a variation in chemical structure as possible. The four negatively solvatochromic (BM, 4PP, ET1, DEMI) and four positively solvatochromic (MV, BDF, PB, DMABR) dyes are shown in - Fig. 2. The only features common to all dyes in the present study is the presence of an electron-donating group $\left(\mathrm{O}^{-}, \mathrm{N}\right.$ or $\left.\mathrm{C}^{-}\right)$, an electron-accepting group $\left(\mathrm{N}^{+}\right.$or $\left.\mathrm{C}=0\right)$ and a conjugated system in between. No other structural features are replicated. Any effects of potency must therefore be due to specific interactions with the dyes' solvatochromic system (ground and/or excited states).

By looking at a range of encapsulated solvatochromic dyes of widely differing structure, covering both positively and negatively solvatochromism, it should, in principle, be possible to discover the common underlying dynamic involved in the interaction between potencies and push-pull molecular systems.

\section{Materials and Methods}

\section{Experimental Protocol}

The experimental protocol is essentially as described previously. ${ }^{3}$ However, some minor changes have been made, as steps employed previously to exhaustively remove leachates from amber moulded glass bottles were deemed unnecessary as studies have progressed. Consequently, on obtaining potency and control solutions in $90 \%$ ethanol from the pharmacy, two sequential 100 -fold dilutions were performed into a final total of $5 \mathrm{~mL}$ reverse osmosis water in ( $8 \mathrm{~mL}$ ) high density polyethylene (HDPE) bottles to give a 10,000 -fold dilution. These bottles were then stored separated by a minimum distance of at least $1.5 \mathrm{~m}$ and used without further processing.

Assays involved taking $50 \mu \mathrm{L}$ of each solution and adding these aliquots to $2.95 \mathrm{~mL}$ of pre-prepared dye/encapsulator solution. Control-dye/encapsulator and potency-dye/ encapsulator solutions were then placed in black film canisters as described previously. ${ }^{2}$ Both control-dye/encapsulator and potency-dye/encapsulator solutions were monitored by scanning against blanks containing buffered encapsulator solution alone at intervals up to a maximum of 5 days. Difference spectra between controls and samples were obtained by instrument subtraction.

The homeopathic potency Arsenicum 10M has been used throughout this study, with additional confirmatory use of Glycerol 50M. This has allowed comparisons to be made between all dyes, both in this study and in previous studies. Results with Glycerol 50M are not reported here, but in all cases confirm the results obtained with Arsenicum 10M.
Arsenicum 10M was chosen because the starting material (arsenic trioxide) can be obtained in very high purity, and the homeopathic literature ${ }^{17}$ lists Arsenicum as fast and long lasting in its clinical action-properties seen as being favourable for, and maximising the ability to detect, physicochemical activity in any chemical-based detection system. A high potency (10M) was chosen for reasons given previously to avoid any mixed material and potency effects possible at low potencies. ${ }^{1}$

\section{Reagents}

MV, DMABR, DEMI, PB, $\beta-C D, \alpha-C D$, citric acid/sodium citrate, sodium dihydrogen phosphate/disodium hydrogen phosphate, boric acid/sodium borate and sodium N-cyclohexyl-3-aminopropanesulfonate (CAPS) were obtained from Sigma Aldrich (UK) and were of the highest purity available.

ET-1 was synthesised and provided by Innovapharm Ltd., Kiev, Ukraine. Structure and purity were confirmed by nuclear magnetic resonance (NMR).

The provenances of $\mathrm{BM}, 4 \mathrm{PP}$ and bis-dimethylamino fuchsone (BDF) were as previously described. ${ }^{1-3}$

$\beta$-CDSBE was obtained from Carbosynth, UK.

Reverse osmosis water was used throughout this study and had a resistivity of $15 \mathrm{M} \Omega \mathrm{cm}$ (checked daily).

Disposable high-purity ultra violet (UV)-transparent cuvettes (Brand $\mathrm{GmbH}$ ) with stoppers were used throughout and are as described previously. ${ }^{1}$

Black film canisters were obtained from Geo-Versand, $\mathrm{GmbH}$.

\section{Dye Solution Storage and Use}

As in previous studies, ${ }^{1-3}$ dye solutions were made and stored in HDPE bottles and allowed to equilibrate overnight before use. All dye solutions were stored in the dark as a precaution against light-induced degradation. This was particularly necessary for the solvatochromic dye DEMI, which is subject to oxidative degradation under the influence of light. However, to ensure compatibility between dyes and with previous studies all dye solutions were treated identically.

Dyes were prepared in buffers at concentrations sufficient to give an absorbance of between 0.5 and 1.5 (generally $50 \mu \mathrm{M})$. Buffer solutions in which dyes were dissolved were at a concentration of $20 \mathrm{mM}$ throughout and at $\mathrm{pH}$ values well above the respective $\mathrm{pK}_{\mathrm{a}}$ values of dyes, for reasons given previously. ${ }^{3}$ Encapsulators $\alpha-C D, \beta-C D$ and $\beta-C D S B E$ were used at a concentration of $10 \mathrm{mM}$.

Light excluding black plastic film canisters were used to store control-dye and potency-dye solutions between scans, as described previously. ${ }^{2}$

\section{Homeopathic Potencies and Control Solutions}

Arsenicum 10M and Glycerol 50M were obtained from Helios Homeopathy Ltd, Tunbridge Wells, UK.

\section{Instrumentation}

UV/visible spectra were recorded on a Shimadzu UV-2600 double-beam spectrophotometer. Buffers were prepared using a Hanna pH210 microprocessor pH meter. 


\section{Results}

Results of incubating Arsenicum 10M with all dye-encapsulator solutions are shown in -Fig. 3A-D for positively solvatochromic dyes and $\mathbf{- F i g}$. 4A-D for negatively solvatochromic dyes. The scans shown are the maximum difference spectra observed, with corresponding times after mixing of potency with dye/encapsulator solutions. Details of dye concentrations, $\mathrm{pH}$ and encapsulator type are given in the figure captions. Spectra are not to scale for the reasons given below but are presented to show the changes accompanying the action of potency.

For the former group, scans (-Fig. 3) show on the left dye/ encapsulator solution (for comparison) and on the right a difference spectrum of potency-dye/encapsulator solution minus control-dye/encapsulator solution. The change in dye spectra on adding potency compared with that of controls is of the order of $2 \%$ with an accompanying $0.4 \mathrm{~nm}$ shift. Consequently, difference spectra are the clearest method of demonstrating what effect potency is having on dye/encapsulator solutions. For all four positively solvatochromic dyes, potency produces a bathochromic (red) shift in the dyes' spectra. - Table 1 provides data on the wavelength changes observed.

For the latter group, scans ( - Fig. 4) show on the right dye/ encapsulator solution (for comparison) and on the left a difference spectrum of potency-dye/encapsulator solution minus control - dye/encapsulator solution. Changes in dye spectra on adding potency compared with that of controls are of the order of $1 \%$ with an accompanying $0.2 \mathrm{~nm}$ shift. What is striking is that for all four negatively solvatochromic dyes potency produces a hypsochromic (blue) shift in the dyes' spectra. - Table 1 provides data on the wavelength changes observed.

Results show the effect of potency is consistent for all positively solvatochromic dyes regardless of structure and consistent for all negatively solvatochromic dyes regardless of structure. Moreover, the effect is opposite and complementary for the two classes of dye. This points to a common underlying mechanism for the action of potency on solvatochromic dyes.
A

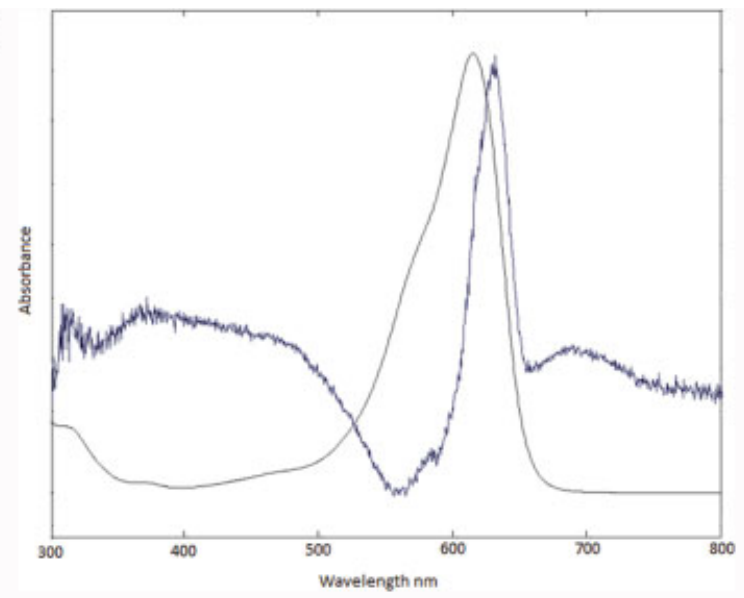

C

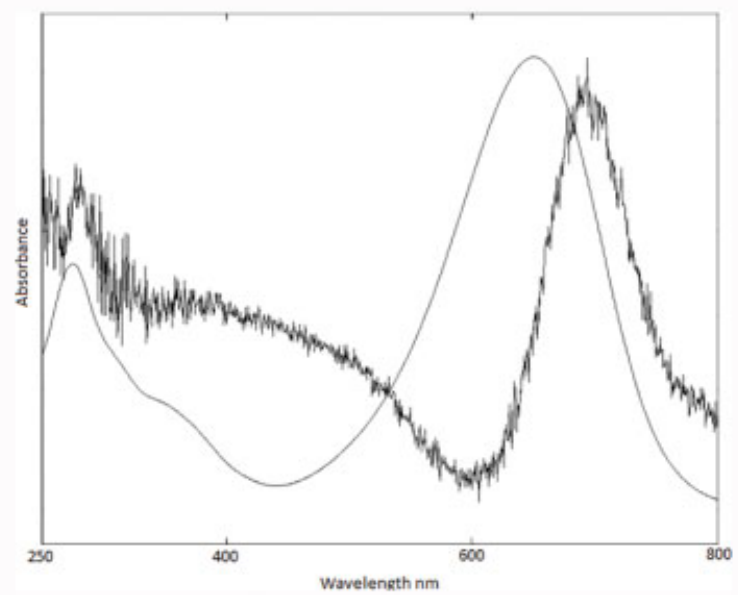

B

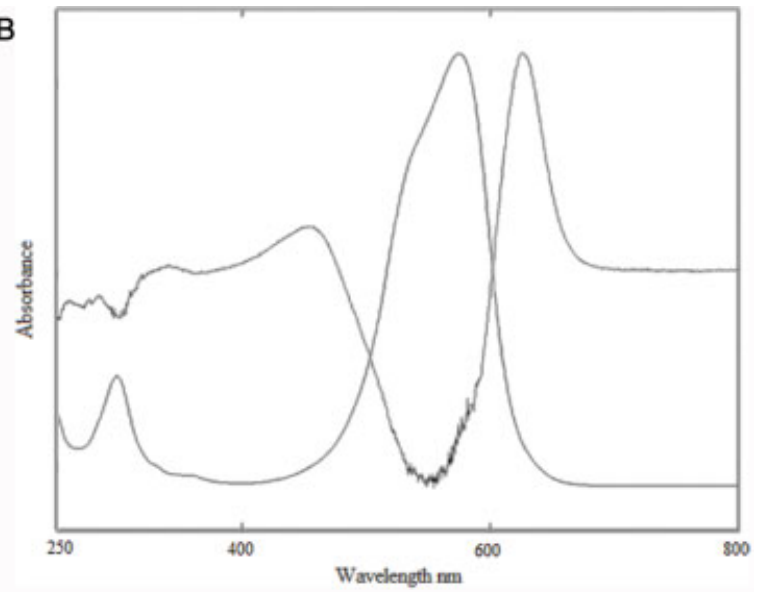

D

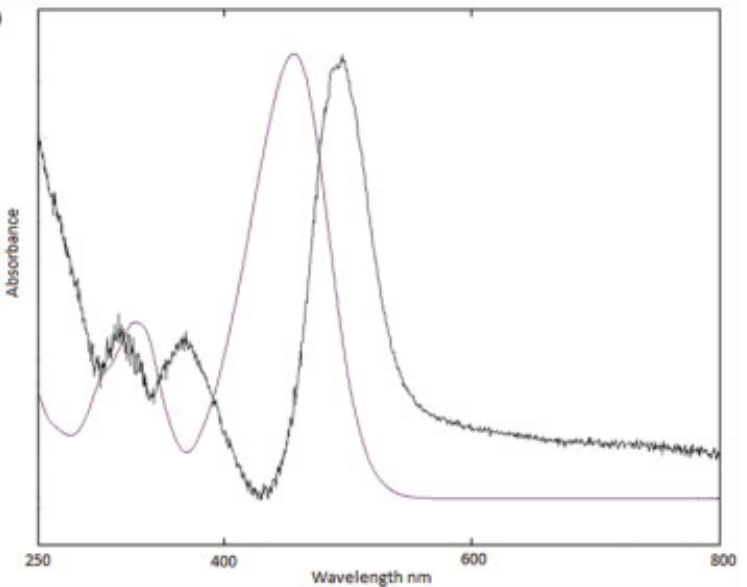

Fig. 3 (A) Spectrum of $50 \mu \mathrm{M} \mathrm{MV} / 10 \mathrm{mM} \beta-\mathrm{CD} / 20 \mathrm{mM}$ phosphate buffer $\mathrm{pH} 7.5$ (left); difference spectrum at $t=3$ days following addition of Arsenicum 10M (right). (B) Spectrum of $50 \mu \mathrm{M}$ BDF/10 mM $\beta$-CDSBE/20 mM borate buffer pH 9.0 (left); difference spectrum at $t=4$ days following addition of Arsenicum 10M (right). (C) Spectrum of $50 \mu \mathrm{M} \mathrm{PB} / 10 \mathrm{mM} \alpha-\mathrm{CD} / 20 \mathrm{mM}$ borate buffer pH 9.0 (left); difference spectrum at $t=12$ hours following addition of Arsenicum 10M (right). (D) Spectrum of $50 \mu \mathrm{M}$ DMABR/10 mM $\beta$-CDSBE/20 mM borate buffer pH 9.0 (left); difference spectrum at $t=50$ minutes following addition of Arsenicum 10M (right). In each case (A, B, C, D), see Materials and Methods for details. BDF, bis-dimethylaminofuchsone; $\beta$-CDSBE, $\beta$-cyclodextrin sulphobutylether; DMABR, dimethylamino-benzylidene-rhodanine; MV, methylene violet; PB, phenol blue. 
A

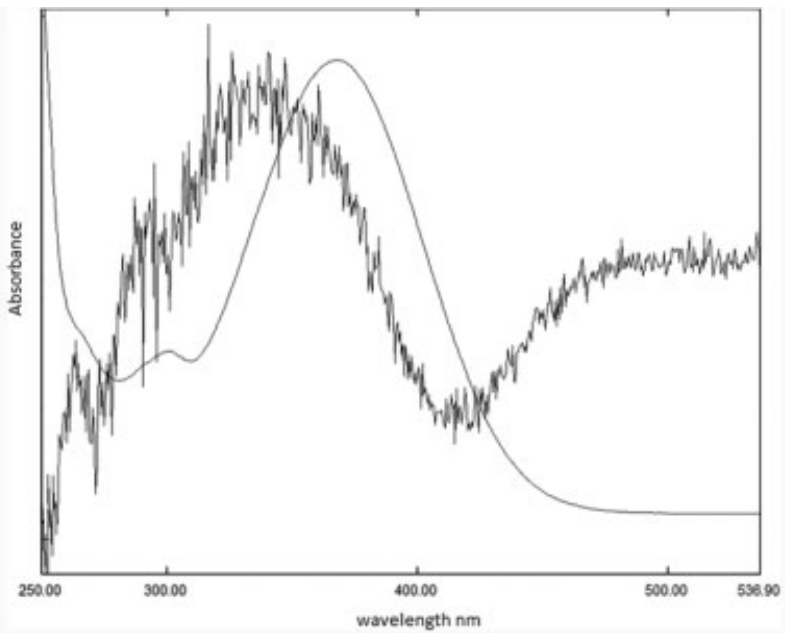

C

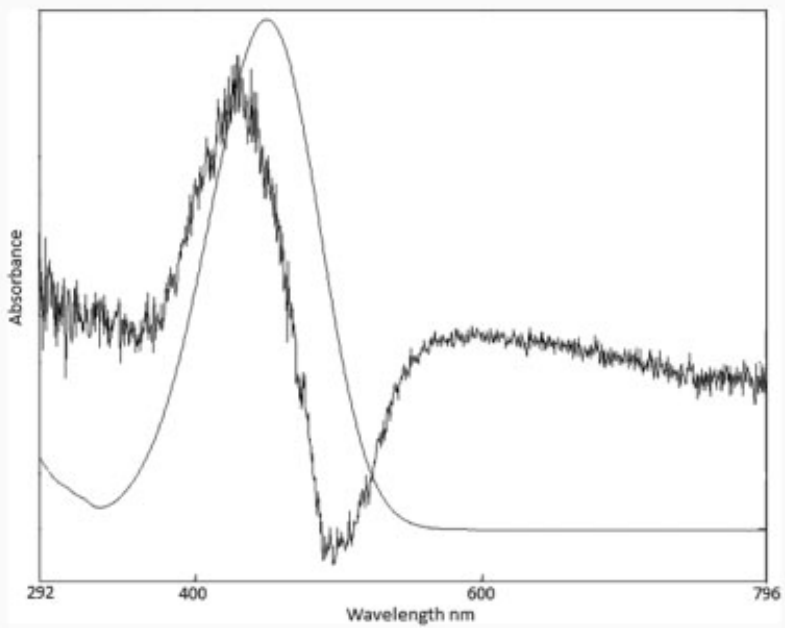

B

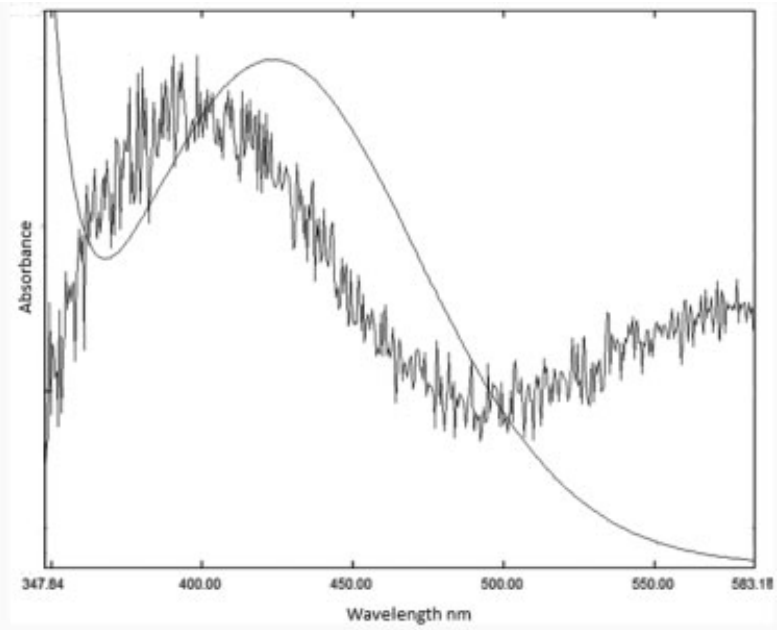

D

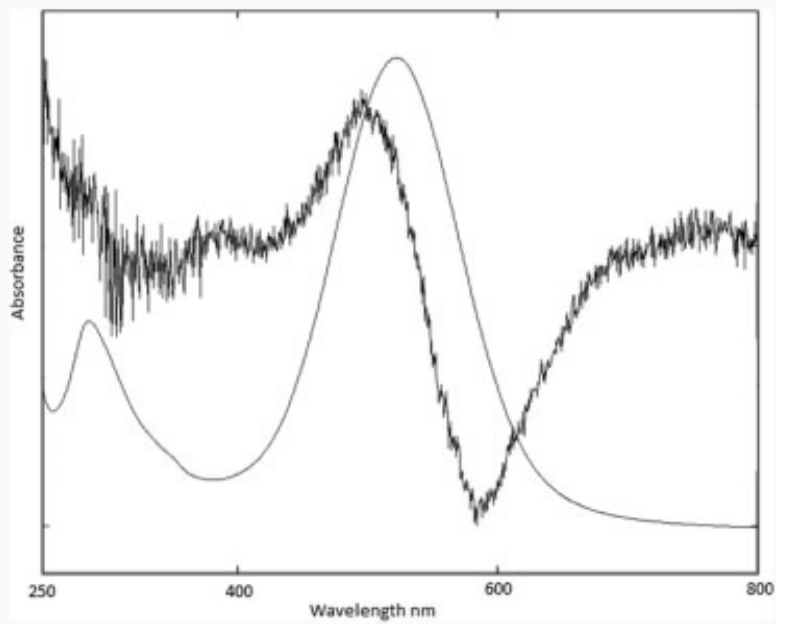

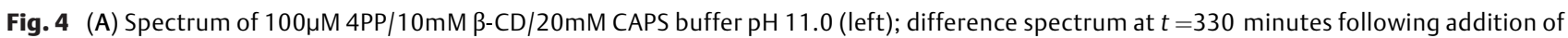

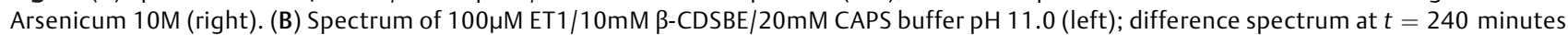
following addition of Arsenicum 10M (right). (C) Spectrum of $50 \mu \mathrm{M} \mathrm{BM} / 10 \mathrm{mM} \beta-\mathrm{CD} / 20 \mathrm{mM}$ CAPS buffer pH 11.0 (left); difference spectrum at $t=12$ hours following addition of Arsenicum 10M (right). (D) Spectrum of $50 \mu \mathrm{M}$ DEMI/10mM $\beta$-CDSBE/20mM phosphate buffer pH 7.0 (left); difference spectrum at $t=230$ minutes following addition of Arsenicum 10M (right). In each case (A, B, C, D), see Materials and Methods for details. 4PP, 4-pyridinium phenolate; $\beta$-CDSBE, $\beta$-cyclodextrin sulphobutylether; CAPS, N-cyclohexyl-3-aminopropanesulfonate; DEMI, cyano-3[4-(dicyanomethyl) phenyl]-2-propenylidene]-N-ethyl-ethaniminium inner salt. ET1, 4-(2,4,6-triphenylpyridinium-1-yl)phenolate; BM, Brooker's merocyanine.

\section{Discussion}

Although a full exposition of the differences between positively and negatively solvatochromic dyes has been given previously, ${ }^{1}$ it is valuable to re-examine the electronic significance of these two types of dyes and the relevance for understanding the nature of their interaction with homeopathic potencies. On irradiation with light of an appropriate wavelength, a solvatochromic dye moves from its electronic ground state to a higher energy electronic excited state together with a spatial movement of electron density along its length-a property known as intra-molecular electron transfer. ${ }^{7,18}$ The closer the ground and excited states are, the less energy is required to produce this transition and consequently the longer the wavelength of light absorbed. Conversely, the further apart the two states are, the more energy is required and the shorter the wavelength of light absorbed. Any environmental factors, agents or conditions that differentially stabilise the ground and excited states-that is, move the states closer together or further apart-will affect the wavelength of light required to produce the electronic transition mentioned above and therefore the absorbance maximum of the dye. Only two agents differentially stabilise ground and excited states in solvatochromic dyes. These are solvent polarity ${ }^{18}$ and electric fields, ${ }^{19,20}$ and consequently solvatochromic dyes are also electrochromic. $^{21-24}$

From - Fig. 5, it can be seen that for negatively solvatochromic dyes an applied electric field will differentially stabilise the dyes' ground (charged) state and therefore shift the energy of light required to cause an electronic transition to shorter wavelengths (hypsochromic shift). For positively solvatochromic dyes, the opposite is true: an applied electric field differentially stabilises the dyes' excited (charged) state, thereby shifting the energy of light required to cause an electronic transition to longer wavelengths (bathochromic shift). 
Table 1 Absorbance maxima of encapsulated dyes together with the changes induced by Arsenicum 10M

\begin{tabular}{|c|c|c|c|c|}
\hline $\begin{array}{l}\text { Dye (with } \\
\text { encapsulator } \\
\text { and } \mathrm{pH} \text { ) }\end{array}$ & $\begin{array}{l}\text { Absorbance } \\
\text { maximum of } \\
\text { encapsulated dye }\end{array}$ & $\begin{array}{l}\text { Change in dye } \\
\text { spectrum on } \\
\text { addition of potency }\end{array}$ & $\begin{array}{l}\text { Spectrum shift: } \\
\text { bathochromic (B) } \\
\text { or hypsochromic }(\mathrm{H})\end{array}$ & $\begin{array}{l}\text { \% change in spectrum }{ }^{a} \text { with } \\
\text { standard deviation (SD) and } \\
\text { number of experiments (n) }\end{array}$ \\
\hline \multicolumn{5}{|c|}{$\begin{array}{l}\text { Positively } \\
\text { solvatochromic dyes }\end{array}$} \\
\hline $\begin{array}{l}\mathrm{MV} / \beta-\mathrm{CD} \\
\mathrm{pH} 7.5\end{array}$ & $615.5 \mathrm{~nm}$ & $\begin{array}{l}\text { Increase at } 628 \mathrm{~nm} \\
\text { Decrease at } 558 \mathrm{~nm}\end{array}$ & $0.5 \mathrm{~nm}(\mathrm{~B})$ & $\begin{array}{l}2.258 \% ; \text { SD } 0.317 \\
n=14\end{array}$ \\
\hline $\begin{array}{l}\mathrm{BDF} / \beta-C D S B E \\
\mathrm{pH} 9.0\end{array}$ & $575 \mathrm{~nm}$ & $\begin{array}{l}\text { Increase at } 626 \mathrm{~nm} \\
\text { Decrease at } 535 \mathrm{~nm}\end{array}$ & $0.4 \mathrm{~nm}(\mathrm{~B})$ & $\begin{array}{l}2.48 \% ; \text { SD } 0.302 \\
n=6\end{array}$ \\
\hline $\begin{array}{l}\mathrm{PB} / \alpha-\mathrm{CD} \\
\mathrm{pH} 9.0\end{array}$ & $650 \mathrm{~nm}$ & $\begin{array}{l}\text { Increase at } 692 \mathrm{~nm} \\
\text { Decrease at } 598 \mathrm{~nm}\end{array}$ & $0.4 \mathrm{~nm}(\mathrm{~B})$ & $\begin{array}{l}0.6 \% ; \text { SD } 0.099 \\
n=4\end{array}$ \\
\hline $\begin{array}{l}\text { DMABR/B-CDSBE } \\
\mathrm{pH} 9.5\end{array}$ & $456 \mathrm{~nm}$ & $\begin{array}{l}\text { Increase at } 494 \mathrm{~nm} \\
\text { Decrease at } 430 \mathrm{~nm}\end{array}$ & 0.25 nm (B) & $\begin{array}{l}1.98 \% ; \text { SD } 0.215 \\
n=7\end{array}$ \\
\hline \multicolumn{5}{|c|}{$\begin{array}{l}\text { Negatively } \\
\text { solvatochromic dyes }\end{array}$} \\
\hline $\begin{array}{l}4 \mathrm{PP} / \beta-\mathrm{CD} \\
\mathrm{pH} 11.0\end{array}$ & 368 nm & $\begin{array}{l}\text { Increase at } 340.5 \mathrm{~nm} \\
\text { Decrease at } 416.5 \mathrm{~nm}\end{array}$ & $0.2 \mathrm{~nm}(\mathrm{H})$ & $\begin{array}{l}0.99 \% ; \text { SD } 0.22 \\
n=4\end{array}$ \\
\hline $\begin{array}{l}\mathrm{BM} / \beta-\mathrm{CD} \\
\mathrm{pH} 11.0\end{array}$ & $449.5 \mathrm{~nm}$ & $\begin{array}{l}\text { Increase at } 431 \mathrm{~nm} \\
\text { Decrease at } 496 \mathrm{~nm}\end{array}$ & $0.2 \mathrm{~nm}(\mathrm{H})$ & $\begin{array}{l}1.038 \% ; \text { SD } 0.25 \\
n=12\end{array}$ \\
\hline $\begin{array}{l}\text { ET1/ } \beta-C D S B E \\
\mathrm{pH} 11.0\end{array}$ & $423.5 \mathrm{~nm}$ & $\begin{array}{l}\text { Increase at } 397 \mathrm{~nm} \\
\text { Decrease at } 492 \mathrm{~nm}\end{array}$ & $0.3 \mathrm{~nm}(\mathrm{H})$ & $\begin{array}{l}0.65 \% ; \text { SD } 0.071 \\
n=6\end{array}$ \\
\hline $\begin{array}{l}\text { DEMI/ } \beta \text {-CDSBE } \\
\mathrm{pH} 7.0\end{array}$ & 522 nm & $\begin{array}{l}\text { Increase at } 498 \mathrm{~nm} \\
\text { Decrease at } 587 \mathrm{~nm}\end{array}$ & $0.2 \mathrm{~nm}(\mathrm{H})$ & $\begin{array}{l}0.96 \% ; \text { SD } 0.14 \\
n=5\end{array}$ \\
\hline
\end{tabular}

Abbreviations: 4PP, 4-pyridinium phenolate; BDF, bis-dimethylaminofuchsone; BM, Brooker's merocyanine; DEMI, cyano-3-[4-(dicyanomethyl) phenyl]-2-propenylidene]-N-ethyl-ethaniminium inner salt; ET1, 4-(2,4,6-triphenylpyridinium-1-yl) phenolate; DMABR, dimethylamino-benzylidenerhodanine; MV, methylene violet; $\mathrm{PB}$, phenol blue; SD, standard deviation.

a\% change in absorbance at the difference absorbance maxima. Absorbance changes in general are lower than those reported previously for free dyes in solution, ${ }^{3}$ reflecting the more stringent conditions involving the use of encapsulators and high $\mathrm{pH}$ values in the current study.
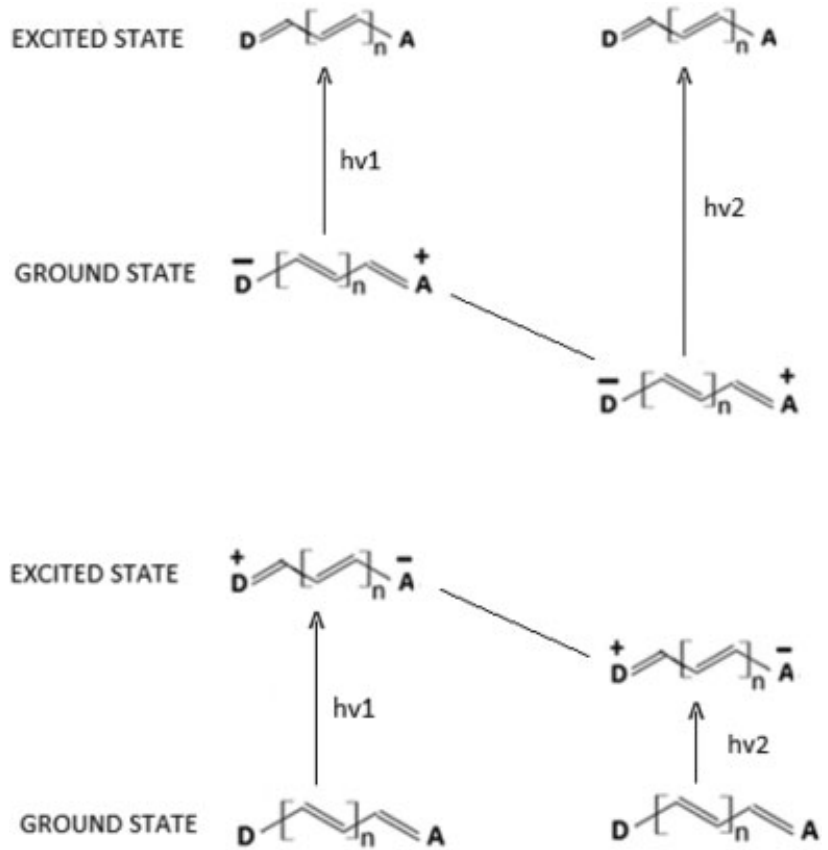

Fig. 5 Representation of the differential stabilisation of the ground state in negatively solvatochromic dyes and consequently higher energy (shorter wavelength) light required for intramolecular charge transfer (upper panel) and the differential stabilisation of the excited state in positively solvatochromic dyes and consequently lower energy (longer wavelength) light required for intramolecular charge transfer (lower panel).
What the results of the current study show is that potencies must be differentially stabilising the ground states of the negatively solvatochromic dyes examined (ET1, BM, 4PP and DEMI) to produce hypsochromic shifts in their spectra and differentially stabilising the excited states of positively solvatochromic dyes examined (BDF, MV, DMABR and PB) to produce bathochromic shifts in their spectra, and that in the absence of a solvent-mediated effect, the presence of an electric field most easily accounts for this.

Before moving on to assess critically the relative merits and consequences of an electric field or solvent-induced differential stabilisation of ground and excited states, it is necessary to look briefly at another potential, though unlikely, explanation for the results seen. It is theoretically possible that the spectral changes observed are due to a change in the binding affinity of dyes for encapsulators. In other words, potency could be causing free (and aggregated) dye to bind more tightly to encapsulator in the case of positively solvatochromic dyes, causing a bathochromic shift, and bound (non-aggregated) dye to bind less tightly in the case of negatively solvatochromic dyes, causing a hypsochromic shift. However, encapsulator binding and aggregation are both sensitive to dye structure, and dyes have been chosen with as large a range of structures as possible. It is unlikely therefore that dyes would collectively respond to potency in the way seen, with positively and negatively solvatochromic dyes binding more and less tightly 
respectively, on the basis of structure. It would be necessary to explain the mechanism by which potencies were causing changes in binding affinity by invoking a change in dye polarity (differential stabilisation of ground and excited states), which is the only dynamic common to all dyes and the very response which can only be explained by the presence of an electric field or a potency-induced change in solvent polarity.

Given that the only explanation for the results in -Figs. 3 and $\mathbf{4}$ is either the presence of an electric field or a change in solvent polarity, one of these two possibilities must be excluded. Potencies must either be acting to increase solvent polarity, which then mediates the spectral changes seen, or potency is acting as an electric field directly on solvatochromic dyes.

There are several lines of evidence that argue strongly against the potency effect being water-mediated and strongly for potencies having an electric field or electric field-like component. These are:

1. Cyclodextrins exclude water from their hydrophobic cores on binding dyes, ${ }^{5}$ so effective solvation of dye by water is prevented. Contact is necessary for a solvatochromic effect, and consequently solvatochromism cannot occur in encapsulated dyes. It is known, however, that imbedding dyes in cell membranes ${ }^{25}$ or in solid matrices ${ }^{23}$ away from solvent does not affect the electrochromic response of push-pull molecular systems.

2. The sensitivity of solvatochromic dyes to homeopathic potencies appears to be a function of dye dipole length and molecular rigidity, ${ }^{3}$ features that do not correlate with solvatochromic response, but do correlate with electrochromic response. ${ }^{26}$

3. Amino acids and dipeptides have their dipole moments increased and electron density distribution affected in electric fields, ${ }^{27,28}$ but they are not solvatochromic. Previous studies have shown $\pi$-bridged amino acids to be sensitive to homeopathic potencies, but not to solvent polarity. $^{3}$

If potencies are exhibiting electric field-like behaviour, several further questions necessarily follow.

The first is: 'Is it possible to assign a figure to the strength of the field?' Using data from Xu et $\mathrm{al}^{24}$ in a study in which the electrochromism of a close analogue of BM was examined in relation to its solvatochromism, together with data from Callis, ${ }^{21}$ it is possible to calculate the strength of an applied electric field necessary to shift the maximum wavelength of the dye by $0.2 \mathrm{~nm}$ from its absorbance maximum at $450 \mathrm{~nm}$ (-Appendix A).

A field strength of $2.25 \times 10^{-5}$ au or $1.16 \times 10^{7} \mathrm{~V} / \mathrm{m}$ emerges from these calculations. For BM, which has a molecular length of $1.2 \mathrm{~nm},{ }^{29}$ this means the strength of the postulated electric field along its length must be $14 \mathrm{mV}$ to account for the results presented above. The figure for positively solvatochromic dyes may be higher as the wavelength shift on adding potency is of the order of $0.4 \mathrm{~nm}$. Unfortunately, no detailed electrochromic studies of positively solvatochromic dyes are available. However, it is unlikely that an upper limit for the strength of the electric field could be greater than $2.32 \times 10^{7} \mathrm{~V} / \mathrm{m}$ or $23 \mathrm{mV} / \mathrm{nm}$ based on the above calculations.

To put these figures into context, the potential difference across the membrane of a typical human neurone is 70 to $80 \mathrm{mV} .{ }^{30}$ Given a membrane thickness of 7.5 to $10 \mathrm{~nm}$, this gives a field strength of 7 to $10 \mathrm{mV} / \mathrm{nm}$. In contrast, the electrostatic field strengths in the active site of enzymes during catalysis, determined by Stark spectroscopy, ${ }^{31}$ are of the order of 1 to $2 \mathrm{~V} / \mathrm{nm}$, and can be as high as $15 \mathrm{~V} / \mathrm{nm}^{32}$

It is possible therefore that an electric field strength of $12 \mathrm{mV} / \mathrm{nm}$ attributable to Arsenicum 10M could have significant biological effects, at least with regard to the physiology of cell membranes and action potentials, although it is difficult to see how direct effects on enzyme catalysis might occur. It is worth noting, however, that an electric field may be only one component of potencies and their strength may also lie in their specificity, as much as the intensity of their electric field. It may also be the case that the stringent conditions of a chemical assay involving solvatochromic dyes do not accurately reflect the strength of potencies in vivo.

It is also worth pointing out that the maximum absorbance changes following addition of potency to dye/encapsulator solutions occur at different times for different dyes (-Figs. 3 and 4), and that positively solvatochromic dyes in general seem to respond more strongly than negatively solvatochromic dyes. Explanations for neither observation -beyond a correlation with dipole moment size $\mathrm{e}^{3}$-can be given at the present time but indicate that potencies may not be simple linearly acting one-vector stimuli, but may be multi-faceted in their actions.

As shown in - Table $\mathbf{1}$ absorbance changes in general are lower than those reported previously for free dyes in solution, ${ }^{3}$ reflecting the more stringent conditions involving the use of encapsulators and high $\mathrm{pH}$ values in the current study. These lower absorbance changes in part are likely to be due to the suppression of dye aggregation which tends to amplify the effect of potency.

The next question relates to the origin of the postulated electric field. To have an electric field one must have charged particles, and those charged particles must be separated. In other words, electric fields by definition have polarity and can only exist and be maintained if the charges producing them are prevented from recombining. If potencies are producing an electric field, they must therefore exist in some metastable state in which charges are kept separate by an as yet unknown mechanism. Furthermore, the charged particles constituting this state would be expected to be sensitive to applied electric and magnetic fields, heat and light. Recombination of charges as a result of heat (thermoluminescence), light (optically stimulated luminescence) and high voltages (electroluminescence) produce photons, and indeed several studies have indicated the emission of photons by potencies under a variety of conditions. ${ }^{33-35}$

Finally, the question must be raised as to whether the results shown are due to an electric field per se or something entirely new that displays electric field-like properties. This 
is a question that simply cannot be answered at present, but serves to underline the necessity to keep an open mind at all stages and not jump to conclusions-an error that could thereby exclude other possibilities.

\section{Conclusions}

Evidence has been presented through the use of cyclodextrin-encapsulated solvatochromic dyes that indicates Arsenicum $10 \mathrm{M}$ possesses an electric field or electric field-like component, which is responsible for causing the shifts in absorbance in these dyes, and that the shifts are in fact electrochromic rather than solvatochromic. It is possible to obtain a figure for the strength of the electric field of $1.16 \times 10^{7} \mathrm{~V} / \mathrm{m}(12 \mathrm{mV} / \mathrm{nm})$ from comparing electrochromic and solvatochromic studies of the dye BM. This figure is approximately the strength of the potential difference across the membrane of a typical human neurone but a fraction of the field strengths measured inside the active site of enzymes during catalysis.

Nevertheless, it is still possible, given the number of unknowns involved, that the electric field or electric fieldlike nature of homeopathic potencies could be responsible for the clinical effects observed in practice, especially any involving changes in membrane potentials. Some of the unknowns may include a possible magnetic field effect accompanying the electric field effect, the probable enhancing effect of potency specificity, a possible field strength difference between in vitro and in vivo, and as yet unrecognised environmental factors, which may deplete the field strength under the conditions of the current study.

Arguments have been presented that indicate the charged particles necessary to produce an electric field must be being kept separate by some as yet unknown mechanism and that potencies must therefore be in some kind of metastable state. If this is the case then a prediction of photon emission under appropriate stimulation by heat, light or high voltages can be made.

Finally, caution demands that one should not necessarily assume that simply because electric field effects are being observed that one is dealing with an electric field. The effects seen may be due to something electric field-like, and the possibility of a completely new phenomenon should be kept open.

\section{Highlights}

- Encapsulation of solvatochromic dyes by $\beta$-cyclodextrins allows the de-coupling of initial dye-potency interactions from any subsequent processes.

- Examination of this primary interaction shows that positively and negatively solvatochromic dyes collectively respond in opposite and complementary ways to Arsenicum $10 \mathrm{M}$ in accordance with the differential stabilisation of their excited and ground electronic states.

- This response can only be explained by concluding that Arsenicum 10M possesses an electric field(-like) component.
- Using solvatochromic and electrochromic data for Brooker's merocyanine, it is possible to assign a value of $1.16 \times 10^{7} \mathrm{~V} / \mathrm{m}$ to the strength of the postulated electric field of Arsenicum 10M.

- Electric fields require separated charges and consequently a prediction can be made that potencies should emit photons under conditions that favour charge recombination.

\section{Funding}

Funding for this work is gratefully acknowledged from Standard Homeopathic Company/Hylands, USA.

\section{Conflict of Interest}

None declared.

\section{References}

1 Cartwright SJ. Solvatochromic dyes detect the presence of homeopathic potencies. Homeopathy 2016;105:55-65

2 Cartwright SJ. Interaction of homeopathic potencies with the water soluble solvatochromic dye bis-dimethylaminofuchsone. Part 1: pH studies. Homeopathy 2017;106:37-46

3 Cartwright SJ. Degree of response to homeopathic potencies correlates with dipole moment size in molecular detectors: implications for understanding the fundamental nature of serially diluted and succussed solutions. Homeopathy 2018; 107:19-31

4 Mota HMG, Aparicio AC, Oliveira LH, et al. Cartwright's method as a physico-chemical marker of Antimonium crudum biological effect. Int J High Dilution Res 2018;17:14

5 Martin Del Ville EM. Cyclodextrins and their uses: a review. Process Biochem 2004;39:1033-1046

6 Arunkumar E, Forbes CC, Smith BD. Improving the properties of organic dyes by molecular encapsulation. Eur J Org Chem 2005: 4051-4059

7 Misra R, Bhattacharyya SP. Intramolecular Charge Transfer: Theory and Applications. Weinheim: Wiley-VCH; 2018

8 Davis F, Higson S. Cyclodextrins. In: Macrocycles: Construction, Chemistry and Nanotechnology Applications. Chichester: Wiley; 2011:190-244

9 Holt JS, Campitella A, Rich A, Young JL. Spectroscopic characterization of the binding and isomerization cycle of Brooker's merocyanine with $\alpha-, \beta-$, and $\gamma$-cyclodextrins. J Incl Phenom 2008; 61:251-258

10 Hirai H, Toshima N, Uenoyama S. Inclusion complex formation of $\gamma$ cyclodextrin. One host-two guest complexation with water-soluble dyes in ground state. Bull Chem Soc Jpn 1985;58:1156-1164

11 Shaikh M, Mohanty J, Sundararajan M, Bhasikuttan AC, Pal H. Supramolecular host-guest interactions of oxazine- 1 dye with $\beta$ and $\gamma$-cyclodextrins: a photophysical and quantum chemical study. J Phys Chem B 2012;116:12450-12459

12 Jara F, Mascayano C, Rezende MC, Tirapegui C, Urzua A. A spectral and molecular dynamics simulation study of $\beta$-cyclodextrin inclusion complexes with solvatochromic dyes derived from barbituric acid. J Incl Phenom Macroc Chem 2006; 54:95-99

13 Zia V, Rajewski RA, Stella VJ. Thermodynamics of binding of neutral molecules to sulfobutyl ether beta-cyclodextrins (SBE-beta-CDs): the effect of total degree of substitution. Pharm Res 2000;17:936-941

14 Zhang G, Shuang S, Dong Z, Dong C, Pan J. Investigation on the inclusion behaviour of neutral red with hydroxypropyl cyclodextrin and sulfobutylether cyclodextrin. Anal Chim Acta 2002; 474:189-195 
15 Saokham P, Muankaew C, Jansook P, Loftsson T. Solubility of cyclodextrins and drug/cyclodextrin complexes. Molecules 2018;23:1161-1176

16 Szablewski M, Thomas PR, Thornton A, et al. Highly dipolar, optically nonlinear adducts of tetracyano-p-quinodimethane: synthesis, physical characterization, and theoretical aspects. J Am Chem Soc 1997;119:3144-3154

17 Bönninghausen CMF. Therapeutic Pocketbook. Munster: Coppenrath'sche Buch- und Kunsthandlung; 1846

18 Reichardt C, Welton T. Solvent effects on the absorption spectra of organic compounds. In: Solvents and Solvent Effects in Organic Chemistry. 4th ed. Weinheim: Wiley-VCH; 2011:365-367

19 Liptay W. Electrochromism and solvatochromism. Angew Chem Int Ed 1969;8:177-188

20 Albert IDL, Marks TJ, Ratner MA. Rational design of molecules with large hyperpolarizabilities: electric field, solvent polarity, and bond length alternation effects on merocyanine dye linear and nonlinear optical properties. J Phys Chem 1996;100: 9714-9725

21 Callis PR. Electrochromism and solvatochromism in fluorescence response of organic dyes: a nanoscopic view. In: Advanced Fluorescence Reporters in Chemistry and Biology. Heidelberg: Springer; 2010:309-330

22 Niedbalska M, Gruda I. Effect of substituents on the solvatochromism of stilbazolium merocyanines. Can J Chem 1990;68:691-695

23 Davidenko NA, Derevyanko NA, Ishchenko AA, Pavlov VA. Effect of electric field on the optical absorption of amorphous molecular semiconductors doped with polymethine dyes. Phys Solid State 2001;43:1426-1432

24 Xu XF, Kahan A, Zilberg S, Haas Y. Photoreactivity of a push-pull merocyanine in static electric fields: a three-state model of isomerization reactions involving conical intersections. J Phys Chem A 2009;113:9779-9791

25 Loew LM. Design and Use of Organic Voltage Sensitive Dyes. In: Canepari M, Zecevic D, eds., Membrane Potential Imaging in the Nervous System: Methods and Applications. New York, NY: Springer; 2011:13-23

26 Miller EW. Small molecule fluorescent voltage indicators for studying membrane potential. Curr Opin Chem Biol 2016; 33:74-80

27 Mazurkiewicz J, Tomasik P. Effect of external electric field upon selected proteogenic amino acids. Adv Nat Sci 2013;6:35-50

28 Mazurkiewicz J, Tomasik P. Effect of external electric field upon selected model dipeptides. Adv Nat Sci 2014;7:6-11

29 Abdel-Halim ST, Awad MK. Absorption, fluorescence, and semiempirical ASED-MO studies on a typical Brooker's merocyanine dye. J Mol Struct 2005;754:16-24

30 Bruce A. Molecular Biology of the Cell. 6th ed. New York, NY: Taylor and Francis; 2015

31 Fried SD, Boxer SG. Electric fields and enzyme catalysis. Annu Rev Biochem 2017;86:387-415

32 Fried SD, Bagchi S, Boxer SG. Extreme electric fields power catalysis in the active site of ketosteroid isomerase. Science 2014;346:1510-1514

33 Lenger K, Bajpai RP, Drexel M. Delayed luminescence of high homeopathic potencies on sugar globuli. Homeopathy 2008; 97:134-140

34 Assumpção R. Electrical impedance and HV plasma images of high dilutions of sodium chloride. Homeopathy 2008;97:129-133

35 Rey L. Can low-temperature thermoluminescence cast light on the nature of ultra-high dilutions? Homeopathy 2007;96:170-174

\section{Appendix A}

The postulated electric field strength of Arsenicum 10M has been arrived at through the following calculations.

For the BM analogue in Xu et al, ${ }^{24} \lambda$ max in methylcyclohexane is at $614 \mathrm{~nm}$ and in acetonitrile at $562 \mathrm{~nm}$. Static field strengths of 0.00192 au and $0.00778 \mathrm{au}$, respectively, applied to the dye produce $\lambda$ maxs at the same wavelengths. Hence, one

can deduce that a field strength change of 0.00586 au causes a shift in absorbance maximum of $52 \mathrm{~nm}$. A $0.2 \mathrm{~nm}$ shift would therefore require a field strength of $2.2538 \times 10^{-5} \mathrm{au}$.

$1 \mathrm{au}=5.142 \times 10^{11} \mathrm{~V} / \mathrm{m}$ or $5.142 \times 10^{5} \mathrm{mV} / \mathrm{nm}$, so $2.2538 \times 10^{-5} \mathrm{au}=1.1589 \times 10^{7} \mathrm{~V} / \mathrm{m}$ or $11.589 \mathrm{mV} / \mathrm{nm}$.

A rounded-up figure gives an approximate value of $1.16 \times 10^{7} \mathrm{~V} / \mathrm{m}$ for the postulated electric field strength of Arsenicum 10M. 